\title{
Research tool patents: tips for facing a "pay up now or litigate" ultimatum
}

\author{
When holders of research tool patents seeking exorbitant license fees threaten litigation, \\ companies need not rush to accept a license without weighing their options.
}

Adda Gogoris and Pamela Ancona

$M$ uch has been written about whether research tool patents should be granted and whether their enforcement should be limited; some limitations have even been imposed ${ }^{1}$. The long debate over these policies, however, is of little practical help to biotechnology and pharmaceutical companies that are threatened with legal action by the owners of research tool patents. Having secured patent claims covering a unique research tool or covering a family of related assays, some patentees seek to coerce an entire industry into accepting exorbitant license terms, including "reachthrough" royalties, based not on the extent to which the company used the patented tool, but rather on the sales of any drug discovered through such use. In some cases, royalty obligations extend well beyond expiration of the patent.

Faced with such demands, a company must assess and weigh, often under considerable time pressure, its exposure to damages, its freedom to engage in future research, the strength of the asserted patent, the cost of taking a license, and the cost and probability of its prevailing in a patent lawsuit.

This article explores some of the ways in which a commercial user of a patented research tool ("R\&DCo") in the United States can react to an aggressive patent enforcement program by a research tool patentee ("ToolCo"). Usually, ToolCo will send R\&DCo (and its competitors as well) what patent litigators term a notice letter, implicitly threatening R\&DCo with patent infringement litigation unless it agrees to a license with terms very lucrative for ToolCo.

First, R\&DCo should not ignore ToolCo's letter, as doing so might escalate ToolCo's aggressive stance. Panic is also unwarranted because, in almost all cases, there will be time for R\&DCo to assess the situation adequately, even if ToolCo has already filed suit.

Adda Gogoris is a member and Pamela Ancona is an associate at Darby \& Darby P.C., 805 Third Avenue, New York, NY 10022 (adda@darbylaw.com).
R\&DCo should remember that, although ToolCo may be prepared to litigate, it will likely not be eager to do so because litigation places at risk the validity of its own patents, which may be ToolCo's only assets.

Second, R\&DCo should realize that it can quickly conduct a preliminary investigation that will permit it to respond to ToolCo's letter in an informed manner. The investigation should include five interrelated parts, all of which-save the last-can proceed in parallel: (1) determining the scope of the asserted patent(s); (2) assessing the company's exposure to damages for past infringement; (3) developing potential defenses; (4) limiting future exposure; and (5) weighing the available options.

\section{Determining the scope of the asserted patent(s)}

This is a determination of whether any of R\&DCo's activities are encompassed by the claims of the asserted research tool patent(s). For example, can the scope of the asserted claims be limited in any way to avoid or limit infringement liability? This involves a detailed legal analysis of the patent (including related patents and patent applications subject to publication under the recently enacted patent statute) and of the record that led to its issuance ("file history") and an interpretation of the patent claims. R\&DCo can find advantage in several recent decisions of the Court of Appeals for the Federal Circuit (which hears all appeals in patent cases) wherein patent claims were limited to what is specifically disclosed in the body of the patent. For example, this court recently interpreted the term "admixture" in a claim to mean a "homogeneous admixture"2, which thus excluded heterogeneous mixtures from the claim. The court found the narrow interpretation consistent with use of the term in the patent specification and in the file history ${ }^{3}$. If available, a similar limiting interpretation of ToolCo's claims can place some or all of R\&DCo's research tool use beyond the scope of the asserted patent.

Additionally, R\&DCo should consider examining file histories of corresponding ToolCo patents in other countries to look for
(1) possible infirmities also present in the US patent but not discernible from the US record alone that could serve to limit the scope of the US claims (or even to invalidate them); and (2) patent activities of ToolCo abroad revealing that ToolCo is probably pursuing additional, broader or different, US claims in patent applications that were filed before the law requiring publication became effective. For example, the breadth of the claims pursued (or obtained) by ToolCo abroad, the tenacity of such pursuit, the existence and record of contested proceedings (e.g., oppositions), the prior documents applied against the claims, and the filing of more than one patent application per research tool per country, can yield valuable information that can potentially undermine ToolCo's US patents, identify other companies targeted by ToolCo, and reveal aspects of ToolCo's patent strategy.

Indeed, patent holders like ToolCo often file successive US patent applications for the purpose of obtaining broader or stronger claims than their already issued US patents, or claims directed to the activities of a particular company they have targeted. They thus attempt to diffuse or eliminate defects in the original patent that would provide target companies, including R\&DCo, with defenses. For example, through such a patent application, ToolCo could try to demonstrate that a relevant prior publication that R\&DCo discovered would not have prevented issuance of the patent. An examination of ToolCo's entire patent portfolio may provide advance warning and allow R\&DCo to take appropriate action.

\section{Assessing exposure}

The next step in R\&DCo's analysis is to assess its exposure to damages liability. For example, suppose ToolCo's patent contains the following assay claim: "A method of determining whether a compound that interacts with receptor $\mathrm{Y}$, is an agonist or antagonist of [the relevant signal transduction pathway], the method comprising steps A through F ...." R\&DCo must then audit its research operations to identify which, if any, assays it has used after issuance of the asserted patent fall within the claim. Depending 
on the size and longevity of R\&DCo's research operations and on the availability of accessible and reliable records that track these activities, this task may be daunting. But by making certain assumptions, R\&DCo can drastically reduce the scope of the task. For example, if ToolCo demands "reachthrough" royalties, R\&DCo need look only for assays used to screen product candidates in active development. If, on the other hand, ToolCo seeks a paid-up license, a sampling rather than a census of the extent to which such assays were used may suffice.

Further, R\&DCo might use quarterly $R \& D$ progress reports to identify project leaders or $R \& D$ managers and interview them rather than ordering a massive notebook review. If the circumstances warrant it, $\mathrm{R} \& \mathrm{DCo}$ can take the position that confirmatory screenings of a compound are excluded by the foregoing assay claim because the compound had been "determined" as a Y antagonist before it was tested by the claimed method.

\section{Developing a defensive strategy}

The usual defenses to a charge of patent infringement are noninfringement and patent invalidity. Whether a noninfringement defense is available depends in significant part on the determinations discussed above. An invalidity defense requires a separate analysis depending on the basis-and there are several bases-for asserting the defense. Here we discuss briefly only two common invalidity defenses, and we touch on the complex subject of sharing information with other companies similarly threatened by ToolCo.

Patent invalidity based on prior art. Even in the absence of litigation, the availability of this defense can substantially strengthen R\&DCo's negotiating position. After assessing the scope of ToolCo's patent claims, R\&DCo should conduct a search of the patent and scientific literature in an effort to discover earlier references ("prior art") that disclose either a research tool identical to one encompassed by ToolCo's claims or one differing from the claims in only an obvious or trivial respect ${ }^{4}$. In particular, $\mathrm{R} \& \mathrm{DCo}$ should search for prior art references that were not considered by the patent examiner during prosecution of ToolCo's patents.

In general, the broader the asserted claims, the greater the probability of finding prior art that describes the claimed research tool. Unfortunately, references disclosing research tools may be difficult to identify because they are often described only in the experimental sections of the technical and patent literature and may not be the main focus of a publication. Therefore, it is important to search full text and broadly.
Expanding the full-text search to less accessible patent and literature collections such as in countries of the former USSR can yield close prior art and is worthwhile. The Japanese Patent Office has been cited as a richer repository of research tool patent applications filed by Japanese companies, than its US or European counterparts ${ }^{5}$. Another potential source of prior art is webbased services that match seekers of specific prior art with persons having knowledge of such prior art ${ }^{6}$.

Patent invalidity based on a deficient patent specification. US patent law ${ }^{7}$ requires that a patent contain a description of the claimed invention sufficient to notify the public that the patentee possessed the entire scope of the invention at the time the patent application was filed ("description requirement") and to enable the skilled artisan to make and use the invention ("enablement requirement"). Again, the broader the claim the more likely it is to be held invalid for noncompliance with these requirements, because more information is needed to satisfy them. Claims presented by ToolCo after its initial application is filed in order to cover activities of a target company are more likely

\section{Even if ToolCo pushes for a quick decision, R\&DCo need not, and should not, give in to the patentee's demands}

to be invalid for insufficient disclosure, because the original specification was not directed to that activity and may not have contemplated it.

The Federal Circuit often invalidates such broad or overreaching claims, especially in biotechnology patents, for noncompliance with the "description" or "enablement" requirement. A patent identifying a material only by a functional property (e.g., "Y antagonist") and/or by a research plan for how to find it or make it, without conveying any concrete knowledge about its structure, fails at least the description requirement ${ }^{8}$. This makes patent claims of the type, "A method for treating disease $\mathrm{X}$ by administering to a patient an effective amount of a compound identified as a Y-receptor antagonist by the method of [the above assay claim]," that call a compound identified solely by the assay used to elucidate its activity vulnerable to a validity attack, unless the patent specification also describes sufficient structural characteristics to show that the patentee was in possession of such compounds.

If there are indications that ToolCo's patents have a weak specification, R\&DCo should invest in a legal analysis to determine the availability and strength of such a defense. This type of defense is attractive not only because it is supported by substantial legal precedent, but also because the defect that gives rise to the defense cannot be cured.

Other defenses. Occasionally, patent invalidity can be established on other grounds such as inequitable conduct ${ }^{9}$ (withholding material information from the patent examiner), or improper inventorship of the patent (someone else made the invention first, or the patentee intentionally appropriated another person's contribution). But these defenses are rarely established from publicly available documents (i.e., before a lawsuit is filed and witnesses are deposed and the patentee's documents become available to the defendant), unless the accused infringer happens to communicate directly with persons having knowledge of such defenses.

Patent misuse (a defense created by judges to stop overreaching practices by the patentee), if established, will render the patent unenforceable, and should be considered if the royalties demanded by the patentee are truly burdensome. Reach-through royalties, especially if they extend beyond expiration of a patent, may be an attractive anchor for a patent misuse defense even though patent misuse has fallen into some disfavor and decisions dealing with this defense are not always consistent.

Sharing information with other accused infringers. Sharing information and coordinating defenses with other accused infringers, even though they may be competitors of R\&DCo, may be attractive because it lowers the cost of gathering information (e.g., by sharing searches and legal analyses of patent scope and validity), and may also lower or disperse litigation expenses (through sharing experts and discovery documents, or establishing a defense fund).

Sharing information may also help to invalidate the patent or result in a narrow interpretation of its claims that may cause them to be easily circumvented. Once a patent is invalidated in one lawsuit, all would-be infringers escape liability. Similarly, if one court interprets the patent claims narrowly (and the claim interpretation is upheld on appeal), it is easier for all accused research tool users to circumvent the claims.

Generally, accused infringers are free to share costs and information about defenses and other weaknesses in the patentee's position. Information sharing can thus significantly diminish the strength of the patentee's position. Collaboration among co-defendants or potential co-defendants, however, involves several risks that should be heeded. First, cer- 
tain collaborative efforts among competitors can constitute a contract or conspiracy in restraint of trade, which is an antitrust violation $^{10}$; examples include agreements to share information about individual communications with the patentee, to inform competitors of agreements made between an accused infringer and the patentee, or to refrain from accepting a license.

Second, revelation to another accused infringer of privileged communications between an accused infringer and its attorney may constitute a waiver of the attorney-client privilege; this means that the patentee can learn the content of these communications through discovery.

Finally, entrusting valuable defense information (such as a potentially invalidating reference) to another accused infringer who reveals it to the patentee prematurely (and thereby gives the patentee the opportunity to "wash" its patent of it, for example, through patent re-examination) or wastes it by using it ineffectively at trial, practically destroys its value for all accused infringers. This is because once a patent has survived a validity challenge, it is very difficult to invalidate the patent based on the same information. It may be preferable for the holder of the defense information to join (intervene) in or otherwise take control of the litigation.

On balance, however, carefully circumscribed communication between co-defendants is beneficial. It is therefore surprising that it is underused and that ToolCo can accuse the entire pharmaceutical industry of infringement without any reports of this activity appearing in the press. Yet, cautiously used, the press has a clear role not only in helping the accused infringers identify one another but also in enlisting public support for their position and in assisting in development of additional defenses.

\section{Limiting future exposure}

A "willful" infringer's liability can be increased up to three times the amount of actual damages. Thus, R\&DCo acts at its own peril if it continues blithely to use assays that even arguably meet ToolCo's patent claims after R\&DCo has received ToolCo's notice letter. If ToolCo prevails and R\&DCo is found to infringe, R\&DCo may be deemed a willful infringer for any use of the patented research tools after being notified of ToolCo's patent.

Among several options that may be available to R\&DCo for limiting its future exposure are the following: (1) obtaining a wellreasoned opinion from patent counsel that the asserted patents are invalid or not infringed. Such an opinion will limit R\&DCo's liability even if it is ultimately found to infringe. (2) Switching to nonin- fringing alternative research tools. It may be possible for R\&DCo to switch to a cell-free assay to avoid use of an infringing cell-based assay. (3) Instituting a "patent watch" to identify issuance of additional ToolCo patents that may be infringed by R\&DCo's activities.

Of course, if these options are unavailable or unsatisfactory, this will influence R\&DCo's decision whether to accept a license, as we discuss below.

\section{Weighing options}

After conducting the foregoing investigation and analysis, R\&DCo will be better equipped to weigh its risks and decide whether to accept a license, negotiate more favorable license terms, go to court, help a competitor litigate against ToolCo (or intervene in that litigation), and/or reposition itself so as to avoid infringement. Even after negotiations are underway, or after a lawsuit has begun, R\&DCo can refine its analysis and revise its conclusion. If the license terms offered by ToolCo are unreasonable, and the patent cannot be avoided but its validity is questionable, litigation, though expensive, may be the preferred alternative.

In conclusion, when a company is accused of infringing a research tool patent, it can proceed diligently but quickly to gather and analyze the information needed to make a proper decision and to develop a defense strategy. Even if ToolCo pushes for a quick decision, R\&DCo need not, and should not, give in to the patentee's demands. By exposing some of the weaknesses in ToolCo's patent, R\&DCo can gain time to assess its position and determine the optimal course to pursue.

\section{Acknowledgments}

The views expressed here are those of the authors and not of the firm or any of its clients. This article is based in part on research by Anna Lokshina

(Yale University Law School, Class of 2003).

1. See 64 Fed. Reg. 246, 72090 (December 23, 1999) for US NIH guidelines that restrict the ability of patentees to derive financial gain from patents directed to research tools developed with NIH funding.

2. Biovail Corp. Int'l. v. Andrx Pharms., Inc., 239 F.3d 1297, 1302 (Fed. Cir. 2001).

3. Ibid.; see also SciMed Life Sys. v. Advanced Cardiovascular Sys., 242 F.3d 1337 (Fed. Cir. 2001); Medtronic, Inc. v. Advanced Cardiovascular Sys., 248 F.3d 1303 (Fed. Cir. 2001).

4. See, e.g., Sibia Neurosciences v. Cadus Pharmaceuticals, 225 F.3d 1349 (Fed. Cir. 2000).

5. Dueholm, K.L. Exp. Opin. Ther. Patents 8, 871-888 (1998).

6. For example, http://www.BountyQuest.com

7. 35 USC § 112, first paragraph.

8. See, e.g., Regents of Univ. of Cal. v. Lilly \& Co., 119 F.3d 1559 (Fed. Cir. 1997).

9. Hoffmann-LaRoche v. Promega Corp., 1999 U.S. Dist. LEXIS 19059 (N.D. CA, 1999).

10. 15 USC §1 (Sherman Act). 